\title{
mFisheries: Lessons in First Cycle Design of a Context-appropriate Mobile Application Suite
}

\author{
Kim Mallalieu, Candice Sankarsingh \\ The University of the West Indies
}

\begin{abstract}
The impact of technological innovation on mobile telephony is evident. The development of mobile web as a platform in particular has benefited from rapid expansion and consistent advances regarding information and communication infrastructure, services and applications. The increasing ubiquity and affordability of mobile devices indicate that the users' expectations are also evolving. Mobile application markets have emerged as a major player to support user experience in meaningful ways, as they often provide a selection of independent programs that cater to the individual's needs and desires. Though most mobile applications have focused on commercial opportunities, there is also interest in those that target human development opportunities. Nevertheless, the question of how the application of a disruptive technology such as the mobile phone can be applied to development in the Caribbean region remains obscure as there is little by way of case studies to craft a formal strategy. The mFisheries project 2009-2012 allowed a team of mobile application designers and developers from The University of the West Indies, St. Augustine to explore the design, development and implementation of a mobile intervention targeting first-time users in the small-scale fisheries sector of Trinidad and Tobago.
\end{abstract}

\section{Introduction}

There is no denying that within the last five years the world has witnessed the rapid growth and development of new and disruptive technologies. Mobile telephony in particular has gained the most ground as is evident by high penetration levels of mobile phones especially among low income earners and traditionally marginalized population clusters. To a large extent, the very promise of mobile technology to "level" an otherwise uneven playing field in terms of access, supply and demand of information and communication services, has attracted the attention of policy and decision makers and leaders.

Caribbean governments do not differ in their determination to reap the benefits of modern ICTs as demonstrated in acclaimed development initiatives involving the use of mobile phones across the globe. Significant proportions of budget expenditure have been dedicated towards building stronger and more robust platforms and infrastructure. While advances in mobile broadband service, such as the recent introduction of $4 \mathrm{G}$ is commendable, many believe that the region is still not in a position to fully enjoy the benefits of ICT. Policy approaches to human capital and capacity building have been recognized as weak [1]. Similarly, pricing of mobile services is proving to be a challenge and has been named as a contributor to the widening of the digital divide among the poor as well as in outlying communities [2].

There is much to learn and understand about the Caribbean context, its population clusters and their corresponding traits in the risk-averse environment that characterizes Caribbean Society. The matter of how a disruptive technology, such as the mobile phone, is applied to indigenous problem spaces and human development needs in the Caribbean region remains obscure. There is very little by way of case studies that allow interested parties to develop an understanding of how the context, as well as the functionality of mobile technology can be adapted to the user's situation. The authors advocate that if the intention of policy makers is to apply mobile technology to social development, then an examination and understanding of the context and the persons involved are critical steps to ensuring meaningful outcomes and long-term commitment.

\section{Development-focused intervention}

mobile

For the purpose of this paper, the authors are concerned with the application of mobile technology within a problem space for a determined cluster within the Small Island Developing State of Trinidad and Tobago. To a large extent, theirs is an investigation rooted in ICT-enabled human development opportunities that may lead to more informed and inclusive policies. In 2009, the smallscale fisheries sector in Trinidad and Tobago was froth with a number of challenges including: price dispersion, crippling monopolies, wastage, unhygienic practices, at-sea vulnerability, financial mismanagement and lack of representation [3][4]. The potential opportunities for a meaningful mobile intervention in small-scale fisheries formed the basis of the mFisheries project of the Caribbean ICT Research Programme, Trinidad and Tobago 
(CIRPTT) at The University of the West Indies, St. Augustine. The project problem space provided an authentic and indigenous context in which to design, develop and test a development-focused mobile intervention aimed at assisting with personal safety and helping to sustain livelihoods by increasing productivity and social inclusion for a poor, marginalized community.

\section{Hypothesis}

The general hypothesis of the mFisheries project was that development challenges occurring in the small-scale fisheries sector could be significantly alleviated by using mobile technology. The project sought to examine possible influencing externalities and to test the following assumptions in support of the hypothesis:

i. $\quad$ Fluency with purpose-designed mobile applications would provide a sense of competency with information and communications facilities that would encourage use of general-purpose information services and feelings of increased social inclusion.

ii. That purpose-designed mobile applications provide improved management of the time and economy of effort in support of small-scale fisheries livelihoods

iii. That purpose-designed mobile applications provide greater convenience when compared to existing practices in the execution of fisheriesrelated work and are easier to use than general communication offerings in support of small-scale fisheries livelihoods

iv. That improvements in time management, economy of effort and convenience would positively impact the livelihoods of small scale fishers

\section{Context-appropriate application design}

The mFisheries suite refers to a collection of context-appropriate productivity programs intended to be used by small-scale fisherfolk in Trinidad. Development-focused mobile solutions for poor and marginalized communities differ in nature and approach to innovative commercially-focused mobile services for the general public. The CIRPTT team, comprising software developers, engineers and social scientists, designed the suite of mobile applications known as "mFisheries", under close and regular consultation with target users.
Though mobile interventions for the poor in some developing countries have successfully relied on Short Message Service (SMS) capabilities, especially in the areas of health and disease management [5], this was not at all a cost-effective option for the Trinidad and Tobago-based exercise. Context is therefore a critical consideration especially when designing an intervention for a specific cluster or group. Different countries or economies are characterized by different m-readiness profiles or propensities defined by their individual access and capacity conditions [6][7]. Further, profiles vary even among population clusters. Investigations revealed that the mobile practices of fisherfolk involved in the small-scale fisheries sector were decisively different from those of non-fisherfolk. The user's actions cannot be isolated from the environment in which they take place, as actions are understood within a context [8]. Because a given context has different social, political and cultural aspects, the pursuit of mobile opportunities requires consideration of the characteristics of individual countries, the investment context and the demands of specific applications. A case was therefore made for the use of mobile phones with data communications and processing capabilities as well as Global Positioning System (GPS) functionality to address important livelihood challenges articulated by Trinidad and Tobago fisher folk [4].

The Agile software methodology proved to be best suited as it allowed the development team to respond to the unpredictability of user adoption and the team's own unfamiliarity with the environment for which they hoped to design an intervention. The Agile approach placed the fisher at the centre of the development process in a participatory methodology. In addition to allowing practices and work to be scaled appropriately, the methodology embraced iterations. In the case of mFisheries, the participatory and iterative nature of the model provided the opportunity for developers and target users to learn with, and from, each other through repeated cycles of contemplation, articulation, exploration, assessment and acceptance.

While the heart of the mFisheries suite was a set of applications that would be installed on mobile phones, supporting software infrastructure was required on the Web to reduce the computational and storage demands on the phones and to manage application functionality even while participating mobile handsets were powered off.

An open-source cloud computing software architecture was selected to facilitate a solution that would be multi-platform, versatile, scalable, affordable, reusable and accessible to a wide crosssection of users and developers. Application components were implemented using open source tools and designed to adhere to $\mathrm{W} 3 \mathrm{C}$ guidelines to ensure accessibility compliance. 


\section{Mobile application suite}

The multi-purposed ICT intervention comprised eight applications as contemplated and rationalized by Mallalieu and Sankarsingh [4]. The overall intervention is summarized in Table 1.

Table 1 Snap Shot of mFisheries Application Suite

\begin{tabular}{|c|c|c|}
\hline $\begin{array}{l}\text { Application } \\
\text { Name }\end{array}$ & $\begin{array}{l}\text { Proposed } \\
\text { Intervention }\end{array}$ & Function \\
\hline \begin{tabular}{l}
\multicolumn{2}{c}{ GFNF } \\
(Got Fish, \\
Need Fish)
\end{tabular} & \multirow[t]{2}{*}{$\begin{array}{c}\text { Virtual } \\
\text { Marketplace }\end{array}$} & $\begin{array}{l}\text {-Allows fisherfolk to } \\
\text { advertise catch } \\
\text { availability to the wider } \\
\text { public } \\
\text {-Allows individuals } \\
\text { desirous of purchasing to } \\
\text { indicate needs based on } \\
\text { species and quantity } \\
\text {-Provides matches \& } \\
\text { facilitates contact } \\
\text { information between } \\
\text { interested parties. }\end{array}$ \\
\hline Prices & & $\begin{array}{l}\text {-Displays the most recent } \\
\text { prices of fish by type and } \\
\text { market from the relevant } \\
\text { Industry authority }\end{array}$ \\
\hline $\begin{array}{l}\quad \text { GPS } \\
\text { (Global } \\
\text { Positioning } \\
\text { System) }\end{array}$ & $\begin{array}{l}\text { At-Sea Safety } \\
\text { Support }\end{array}$ & $\begin{array}{l}\text { - Provides a periodic } \\
\text { recorded presence (date } \\
\text { and time-stamped } \\
\text { location coordinates) of } \\
\text { the registered user to a } \\
\text { Web server }\end{array}$ \\
\hline S.O.S. & $\begin{array}{l}\text { Emergency } \\
\text { Response } \\
\text { System }\end{array}$ & $\begin{array}{l}\text {-Sends immediate pre- } \\
\text { defined notifications (via } \\
\text { text messaging, email) } \\
\text { and automatically } \\
\text { initiates a voice call to } \\
\text { the Trinidad and Tobago } \\
\text { Coast Guard. }\end{array}$ \\
\hline Compass & $\begin{array}{l}\text { Navigational } \\
\text { Aid }\end{array}$ & $\begin{array}{l}\text {-Personal navigational } \\
\text { tool }\end{array}$ \\
\hline Tips & & $\begin{array}{l}\text {-Audio podcasts on } \\
\text { fisheries-related themes }\end{array}$ \\
\hline $\begin{array}{l}\text { First Aid } \\
\text { Training } \\
\text { Companion }\end{array}$ & $\begin{array}{l}\text { Audio-visual } \\
\text { Training }\end{array}$ & $\begin{array}{l}\text {-Short Audio-visual } \\
\text { training videos on } \\
\text { attending to common } \\
\text { fisheries-related injuries }\end{array}$ \\
\hline Camera Tool & $\begin{array}{c}\text { Photo } \\
\text { Journalism }\end{array}$ & $\begin{array}{l}\text { - Allows fisherfolk to } \\
\text { report injustices/ } \\
\text { grievances by uploading } \\
\text { photo evidence to an } \\
\text { online database. }\end{array}$ \\
\hline Info Zone & $\begin{array}{l}\text { Information } \\
\text { facilities and } \\
\text { services }\end{array}$ & $\begin{array}{l}\text {-Survey, feedback } \\
\text { facilities and links to } \\
\text { fisheries-related } \\
\text { information }\end{array}$ \\
\hline
\end{tabular}

i. Virtual Market Place

The Virtual Market Place is comprised of two component applications: Got Fish Need Fish (GFNF) and Prices. Prices displays the most recent prices of fish in the Port of Spain and Orange Valley markets, as determined by the National Agricultural
Marketing and Development Corporation (NAMDEVCO). GFNF is an interactive application which facilitates the broadcast of notifications from individuals who have fish for sale (Got Fish) and those who wish to purchase fish (Need Fish). It facilitates a match between these two parties based on the advertised species, pricing and quantities.

\section{ii. At-Sea Safety and Navigation}

The At-Sea Safety and Navigation utilities comprise four components: SOS, Compass, GPS and at-sea tracking. SOS, the commonly used description for the international morse code distress signal, sends immediate pre-defined notifications in the form of email and text messages and automatically initiates a voice call to the Trinidad and Tobago Coast Guard (TTCG) when initiated by a user. Compass provides the traditional features of a dedicated compass on the interactive mobile screen. GPS logging and retrieval facilitate user-controlled storage and access of GPS points. At-Sea Tracking is a position tracking system which periodically records, on a Web server, the date and time stamped location coordinates of participating fisher folk at sea. This tracking is triggered when the mobile phone is detected as having left the geofence defined by the land boundaries of Trinidad or Tobago.

\section{iii. Training Support}

Training Support comprises two components: First Aid Training Companions and Tips. First Aid Training Companions are multimedia training modules adapted from curriculum developed by the Caribbean Fisheries Training and Development Institute (CFTDI). Oral instructions are synchronised with text and images to describe the steps to be taken in case of various emergency scenarios. Multimedia controls for stopping, pausing and playing the training content are included. Tips delivers locallydeveloped audio podcasts on themes such as emergency maintenance, fishing methods, handling of fish, preparation for sea, rules of the road and survival at sea.

\section{iv. Citizen Participation}

Facilities for citizen participation have been included in the mFisheries suite in the form of a Camera tool with supporting data logging and upload facilities. Photographs taken by fisherfolk are shared via web-based Google album linked to the mFisheries project website, and mostly feature pictures of prized fish catches.

\section{v. Info Zone}

An addition to the original suite of mFisheries applications was an information zone as a channel through which users may easily access fisheriesrelated information on the Web. The Info Zone also very importantly includes an "mTellUs" mobile 
survey tool that is used by the mFisheries team to solicit and acquire structured data from application users; and a "Feedback" facility for providing unstructured, unsolicited feedback from users to the mFisheries team.

\section{Deployment}

Orientation training and subsidies were deemed necessary to engage and empower users. Rather than donate free smartphone handsets to the target group, a financial commitment of TT\$ 500. was required from interested fisherfolk in order to receive the instrument of an estimated market value of US\$ 400/ TT\$ 2,500. Fisherfolk were responsible for their voice communication charges, however no data charges were applied by the participating service provider in order to specifically access the mFisheries mobile application suite. The approach would not prove to be unreasonable for participation and 52 handsets were deployed to interested fisherfolk at various communities across the islands at the start of the trial in March 2011. The handset itself was configured by development engineers who removed the pre-existing browser applications to prevent misuse of the complimentary data plan, and installed the suite prior to distribution.

Over a period of a year, fortnightly calls were made to each participant for inputs into the ongoing agile development cycle, to collect anecdotal data, to schedule and coordinate visits or to discuss any arising issues. These calls were supplemented by regular field visits and quarterly meetings. From the beginning of the programme, field trial participants were provided with a direct hotline to the deployment team to be used in the event of problems associated with the use of the smartphone and/or the mFisheries suite.

\section{Usage data}

Four applications namely: GFNF, Prices, S.O.S and Camera, communicated directly with the cloud platform, and allowed numerical usage data to be recorded to a database on the Web. Other applications such as: Compass, First Aid, and Tips either relied on content stored locally on the instrument's memory, or in the case of GPS on communication with larger service systems (GPS satellite), and were used at each fisher's discretion and convenience. Anecdotal data was recorded for the latter. The frequency of application usage for the latter was based on self-reporting as the pilot system was unable to monitor individual usage at that level. Later on, a mobile application was provided to facilitate ad hoc feedback. Usage data for applications such as GFNF and Prices was tracked and monitored via the application server. On this basis, 9 participants whose usage was deemed to be unacceptably low were withdrawn from the pilot.

As with any research involving the acceptance and diffusion of an innovation among end-users, there was the expectation that some members of the fishing communities would demonstrate a preference for traditional or manual practices and not be inclined to use new technologies. In recognition of this, the exercise targeted fisherfolk classified as "early adopters" who envisioned value in the intervention at the proposition stage and who were eager to engage with the applications.

It was also anticipated that usage data for the periods under observation would vary with the different categories of fisherfolk participating in the field trial and the extended field trial. Participating vendors and processors, for example, do not go to sea and therefore, as expected, did not use the at-sea applications. Many participating fisherfolk who go to sea do not sell fish so they did not use GFNF, though many did use Prices. Yet still, the relationship between days spent at sea and days spent on land over the period showed irregular at-sea activity.

\section{Evaluating mFisheries: user perceptions}

In recognition of the scope of mFisheries that is clearly within its first development cycle, the authors' evaluation is primarily concerned with users' perception of the applications as well as factors that might lead to the acceptance and adoption, or conversely the rejection, of the intervention into fisheries activities. User acceptance is often the pivotal factor determining the success or failure of an ICT intervention [9]. Likewise, innovation diffusion theory recognizes that while the technical attributes of the innovation may not be significant, perceptions of technology do matter and are important factors influencing technology adoption [10]. Within the limitations of the small sample size and dependence on self-reporting by fisherfolk, the authors have attempted to provide rankings of the user perceptions of the mobile applications in two matrices.

\section{User Acceptance}

The first matrix in Table 2 is concerned with user acceptance. It maps determinants of perceived usefulness from the extended technology acceptance model [11] to Likert scale results. The average of each application has been ranked according to the degree to which it is perceived as either high, medium or low. Four determinants were selected to assess user acceptance of mFisheries. They have been applied independently in each instance and are defined as follows: 
i. job relevance refers to the individual's perception regarding the degree to which the target system is applicable to his job

ii. output quality refers to the individual's perception regarding how well the system performs the tasks

iii. result demonstrability refers to the individual's perception regarding the degree to which the results are tangible

iv. ease of use refers to the degree to which an individual believes that using a particular system would be free of physical and mental effort.

Table 2. User Perceptions: Extended Technology Acceptance

\begin{tabular}{|c|c|c|c|c|}
\hline $\begin{array}{l}\text { User } \\
\text { Perceptions } \\
\text { MATRIX! }\end{array}$ & JobRelerance & Output Quality & $\begin{array}{l}\text { Result } \\
\text { Demonstrability }\end{array}$ & Ease of tise \\
\hline High & $\begin{array}{l}\text { - GANF } \\
\text { - Prices } \\
\text { - S.OS } \\
\text { - Compass }\end{array}$ & $\begin{array}{l}\text { - Prices } \\
\text { - S.OS. } \\
\text { - Camera } \\
\text { - Training } \\
\text { Companions } \\
\text { - Tips } \\
\end{array}$ & $\begin{array}{l}\text { - Prices } \\
\text { - S.OS } \\
\text { - Training } \\
\text { Companions } \\
\text { - Tips }\end{array}$ & $\begin{array}{l}\text { - Prices } \\
\text { - S.OS } \\
\text { - Camera } \\
\text { - Tips }\end{array}$ \\
\hline Medium & $\begin{array}{l}\text { - Training } \\
\text { Companions } \\
\text { - GPS } \\
\text { - Info Zone }\end{array}$ & $\begin{array}{l}\text { - Compass } \\
\text { - GPS } \\
\text { - Info Zone }\end{array}$ & $\begin{array}{l}\text { - Camera } \\
\text { - Compass } \\
\text { - Info Zone }\end{array}$ & $\begin{array}{l}\text { - Compass } \\
\text { - Training } \\
\text { Companions } \\
\text { - Info Zone }\end{array}$ \\
\hline Low & $\begin{array}{l}\text { - Tips } \\
\text { - Camera }\end{array}$ & - GANF & $\begin{array}{l}\text { - GREF } \\
\text { - GPS }\end{array}$ & $\begin{array}{l}\text { - GPS } \\
\text { - GANF }\end{array}$ \\
\hline
\end{tabular}

The two mobile applications that demonstrate high levels of acceptance from the fisherfolk community in all four areas are Prices and SOS. They are both highly perceived as i) being applicable to the work of the fisherfolk, ii) as having fulfilled the task expected by the user and his express needs (spontaneous demand of market prices, ad hoc appeals for at-sea assistance), iii) as supplying results with which fisherfolk could relate, easily identify and thus value, and iv) as being easy for first-time users to operate when compared with other applications contained in the suite. Usage data corroborated with the high rankings of Prices and SOS as fisherfolk used their mobile devices to assist in the sale of their produce and to call for help more than any other fisheries related activity. As ICTenabled interventions, Prices and SOS successfully addressed two of the group's major preoccupations: price dispersion and at-sea vulnerability and mapped them to high-level priorities defined by the users themselves: sale of fish catches and personal safety.

Perceptions regarding Got Fish Need Fish are also interesting. The application's core purpose, that is, the provision of an alternative "point of sale" via a mobile marketplace, was recognized as being very relevant to small-scale fisheries by fisherfolk and the use of the mobile application manageable. However, the application's reliance on an active ecosystem spanning the full small-scale fisheries value chain, outside of the scope of the field trial, constrained its usefulness in three decisive areas. Fisherfolk recognized the added complexity of this application required increased user input. They eventually overlooked the application as it was time-consuming and the scope of the field trial did not accommodate engagement of a critical mass of agents higher up the value chain.

User perceptions of the Compass tool available on the mobile device proved to be satisfactory. The functionality of the application itself relied on the operating system and software on the mobile device as opposed to a traditional and familiar hand-held compass. Though technical issues were minimal, challenges of "unresponsive screens", "time outs" and "delays" especially while at sea, impacted fisherfolk perceptions on the output quality, results and the ease of use of Compass. Interestingly, it was better received than another related application, GPS, which was perceived as being very difficult to use, and with some fisherfolk doubtful of its immediate value to their activities. This has signaled a level of unfamiliarity and lack of fluency with the use of global positioning systems by the majority of smallscale fishermen.

With regards to Training and Tips, user perceptions indicate that the design of these instructional materials were generally relevant, of an impressive quality and easy to use by the fisherfolk. Although perceptions indicate that the multimedia training modules addressed the context of their operations, it is noted that fisherfolk did not rank training as high with regards to job relevance as they did for applications more readily associated with income-earning or at-sea safety. They were also generally unimpressed by the limited and static nature of the training content and pressed for additional content, with a keen interest in receiving new content over time.

The current implementation of the Info Zone provides a limited number of static links to Web resources of relevance to small-scale fisheries. While the mFisheries users were very enthusiastic about this information on first access, subsequent interest dwindled, suggesting that they would highly value dynamic content. The mobile survey tool, mTellUs, was not designed to provide direct benefit to the fisherfolk, but rather to facilitate data gathering for the research team. Nevertheless, it was interesting to note that users were comfortable completing surveys on the mobile phone on request. More interestingly was the use of the unsolicited feedback facility, through which several users sent alerts to the research team regarding the status of various mFisheries matters such as the availability of GPS or cellular service. In some cases full sentences were 
composed using the limited facilities of the mobile keypad.

\section{User Adoption}

The matrix in Table 3 seeks to classify fisherfolk perceptions to explain differences in adoption rates. It maps perceptual characteristics explained by Innovation Diffusion Theory [12] to Likert scale results. The average of each application has been ranked according to the degree to which it is perceived as either high, medium or low. Three of the five perceptual characteristics were selected and again applied independently to each mobile application. They are defined as follows:

i. relative advantage as the degree to which an innovation is perceived as better than the idea it supersedes

ii. compatibility as the degree to which an innovation is perceived as being consistent with the existing values, past experiences and needs of potential adopters

iii. complexity as the degree to which an innovation is perceived as difficult to understand and to use.

Table 3. User Perceptions: Innovation Diffusion Theory

\begin{tabular}{|c|c|c|c|}
\hline $\begin{array}{l}\text { User Perceptions } \\
\text { Matrix 2 }\end{array}$ & Relative.Adrantage (RA) & Compatibility & Complexity \\
\hline High & $\begin{array}{l}\text { Pices } \\
\text { s.o.S. } \\
\text { Compass } \\
\text { GNPF } \\
\text { GPS }\end{array}$ & $\begin{array}{l}\text { Pices } \\
\text { S.OS.S. } \\
\text { Compass }\end{array}$ & GRF \\
\hline Medium & $\begin{array}{l}\text { Training } \\
\text { Tips } \\
\text { Info Zone }\end{array}$ & $\begin{array}{l}\text { Training } \\
\text { Tips } \\
\text { GPS } \\
\text { GNNF } \\
\text { Info Zone } \\
\end{array}$ & $\begin{array}{l}\text { GPS } \\
\text { Info Zone }\end{array}$ \\
\hline Low & & & \begin{tabular}{|l|} 
Prices \\
S.OSS \\
Compass \\
Tips \\
Training \\
\end{tabular} \\
\hline
\end{tabular}

User perceptions regarding the degree to which various mobile interventions superseded prior livelihood conditions and practices demonstrate that fisherfolk highly valued the convenience, as well as context-appropriateness of the mFisheries suite. Prices, as an application that pushed current market prices directly to the handsets of fisherfolk, removed the inconvenience of infrequent announcements at their local depots, searching for the information or relying on word-of-mouth before engaging in sales. Similarly, GFNF, as a promise of a mobile marketplace that could allow greater guarantees and personal control of the sale and purchase of fish, continued to be perceived as being better than the current ad hoc practices that have created crippling monopolies, wastage and price dispersion [4]. In terms of adoption, the essence of GFNF as a space for the trading of goods seems to have been quite compatible with the existing values and needs of the fisherfolk. The concept of a mobile market however presents a new and unfamiliar platform for activity. The high degree of complexity of the enabling social infrastructure indicates that a meaningful trial of GFNF would require richer engagement of agents in the small-scale fisheries value chain.

In the case of SOS, fisherfolk were not only able to initiate a call for help without difficulty but also witnessed in all cases the immediate response of the Trinidad and Tobago Coast Guard since the implementation of this intervention. The high rankings as well as anecdotal data gathered from the users indicate improvements to a previously strained relationship. Further, many fisherfolk expressed appreciation for having a series of productivity programs in one central device. Compass was one such example of this and ranked highly in relative advantage. It also ranked highly with regards to compatibility and supports the case for contextappropriate mobile applications that mimic familiar and traditional hand-held instruments, as the matrix demonstrates it as being readily adopted by the user group. Similarly, applications such as Training and Tips were shown to have superseded face-to-face training sessions that were generally poorly attended by fisherfolk in the past. The use of the Feedback facility in Info Zone indicates that some participants are willing to take the initiative in an agile development team and to compose narrative statements on a hand held device. It also suggests that these users perceive the mobile phone as a legitimate channel for communicating in ways other than voice. In addition to the added convenience of mobile learning, findings confirm that contextappropriate training applications that address the values, experiences and needs of users are likely to be adopted.

\section{Implications of user acceptance and adoption data}

Performance impacts are lost when systems are rejected by users [7]. As a development-focused mobile application suite in its first phase of the software life cycle, there is enough evidence to prove that this has not been the case with mFisheries. Field trial participants have agreed to pay for and manage the data service on the subsidized handsets following the trial period and have demonstrated an eagerness to continue using the smartphone device which they now view as necessary for their livelihood. The tangible expression of willingness by the community that the intervention can exist in harmony with its needs, as well as evidence of a strong desire to 
pursue the intervention rather than revert to prior practice, are both significant outcomes at this stage.

Far from being rejected, it is now evident that there are various classes of mobile applications existing within the suite that require varying levels of knowledge, experience and fluency with the device as well as its supporting technology. The authors believe that the suite itself is distributed between three classes of mobile interventions. The first class is made evident by applications such as Prices, SOS, Tips and Training, Weather Bug \& Tide. These programs in the suite were able to provide instant gratification to the user with minimal inputs for needs-based information. Similarly, applications within this stage rely on a very basic fluency and familiarity with the device and acceptance of the instrument within the physical activities of the fisherfolk. They were among those most used in the first development cycle and scope of the mFisheries exercise.

The second type of application progresses from this to a more in-depth, multi-step engagement with an initiated program. These include GPS logging and retrieval of locations and the Camera tool which all require a sequence of finite and deliberate actions. These types of interventions have necessarily benefited from the user's fluency and success with the first class of applications. As such the user can engage in additional cognitive processes and perform novel tasks related to his livelihood and daily activities with the device.

A third type of application, by example of GFNF, further distinguishes itself from these prior classes of applications with an added layer of complexity requiring the formalization of a wider ecosystem. This class of application is best poised for direct long-term development impacts.

\section{Conclusion}

Technology as any tool serves the purpose of enquiry, enabling people to address problems in context and to clarify and transform them into new understanding [13]. Mobile devices are especially well-suited to context-aware applications [14] as the supporting technology allows context and functionality to be adapted to the user's situation. It appears however that even though the mFisheries suite is context-appropriate, some applications fall outside of the scope of first-cycle deployment, as well as the digital literacy profile of fisherfolk at this point in time.

The versatility of mobile technology however allows context and functionality to be continuously and more frequently adapted to the users' situations. It is believed that the distributed applications will function optimally at different development cycles over time. The lifespan of the first cycle of the mFisheries project, reported here, has afforded the opportunity to operationalize the application suite components across all applicable users while making important observations for the future iterations. Further, the authors believe that further assumptions expressed within their general hypothesis on purpose-designed mobile applications can only be tested and reasonably assessed when the mFisheries suite has benefited from the operationalization of second and third development cycles that cater to more advanced categories of applications.

\section{References}

[1] Sumitra, D. \& Bilbao-Osorio, B. (Eds.). (2012). The global information technology report 2012: Living in a hyperconnected world. Geneva: SROKundig.

[2] Maurius, M. (2012, July 4). Will 4G change mobile broadband in the Caribbean? Retrieved from http://www.ict-pulse.com/2012/07/will-4g-changemobile-broadband-in-the-caribbean/

[3] Mohammed, E., Ferreira, L., Soomai, S., Martin, L. and Chan A. Shing, C. (2011). Coastal fisheries of Trinidad and Tobago. In S. Salas, R. Chuenpagdee, A. Charles and J.C. Seijo (eds). Coastal fisheries of Latin America and the Caribbean. FAO Fisheries and Aquaculture Technical Paper, 544, 315-356.

[4] Mallalieu, K. \& Sankarsingh, C. (forthcoming, 2012). Contemplating Mobile Applications for Small-Scale Fisheries in Trinidad and Tobago. In Dunn, H. (Ed.) Ringtones of Opportunity: Policy, Technology and Access in Caribbean Communications. Kingston: Ian Randle Publishers.

[5] Maparura, S. (2010, December 2nd). An SMS a day keeps the doctor away: The use of mobile phones to improve African health care outcomes. Consultancy Africa Intelligence. Retrieved from: http://www.consultancyafrica.com/index.php?option =com_content\&view=article\&id=615:an-sms-a-daykeeps-the-doctor-away-the-use-of-mobile-phones-toimprove-african-health-care-

outcomes\&catid=57:africa-watch-discussionpapers\&Itemid=263

[6] Maugis, V. ,Choucri, N. , Madnick, S. T. , Siegel, M. D. , Gillett, S. E. , Haghseta, F. , Zhu, H. \& Best, M. L. (2005). Global e-Readiness-for What?Readiness for e-Banking. Information Technology for Development, 11(4), 313-342.

[7] Mechael, P., Batavia, H., Kaonga, N., Searle, S., Kwan, A., Goldberger, A., Fu, L., and Ossman, J. (2010). Barriers and Gaps Affecting mHealth in Low and Middle Income Countries: Policy White Paper. The Earth Institute Columbia University; mHealth 


\begin{abstract}
Alliance.pp. $\quad 79 . \quad$ Retrieved from http://mobileactive.org/research/barriers-and-gapsaffecting-mhealth-low-and-middle-incomecountries-policy-white-paper
\end{abstract}

[8] Uden, L. (2007). Activity theory for designing mobile learning.International Journal of Mobile learning and organisation, 10 (10) 81-102.

[9] Davis, F. (1993). User acceptance of information technology: system characteristics, user perceptions and behavioural impacts. International Journal ManMachine Studies, 38, 475-487.

[10] Jurison, J. (2000). Perceived value and technology adoption across four end-user groups.Journal of End-User Computing, 12 (4), 2128.

[11] Venkatesh, V., \& Davis, F. D. (2000). A Theoretical Extension of the Technology Acceptance Model: Four Longitudinal Field Studies. Management Science, 46(2), 186-204.

[12] Rogers, M. (1983). Diffusion of Innovations. New York: Free Press

[13] Sharples, M., Taylor, J., \&Vavoula, G. (2007). A theory of learning for the mobile age. In R. Andrews \& C. Haythornthwaite (Eds.), The Sage handbook of elearning research (pp. 221-241). London: Sage.

[14] Naismith, L., \&Corlett, D. (2007). Reflections on success: A retrospective of the mlearning conference series 2002-2005. Birmingham, United Kingdom: University of Birmingham, Centre for learning, innovation and collaboration. 